\title{
Lipedema: más que un problema de "piernas gordas". Actualización en la fisiopatología, diagnóstico y tratamiento quirúrgico
}

\author{
Nicolás Pereira C..$^{1,2}$
}

Departamento de Cirugía Plástica, Clínica Las Condes. Santiago, Chile.

2Departamento de Cirugía Plástica y Quemados, Hospital del Trabajador. Santiago,

Recibido 2020-06-30 y aceptado 2020-09-21

Correspondencia a:

Dr. Nicolás Pereira $C$ npereira@clinicalascondes.cl

\section{Lipedema: more than a problem of "fat legs". Update in the pathophysiology, diagnosis and surgical treatment}

Lipedema is a common, frequently under-diagnosed, chronic and progressive disease that generates an important detriment in quality of life. It consists in an abnormal deposit of subcutaneous adipose tissue mainly in the lower extremities, almost exclusively affecting women. Its main symptoms are pain, sensitivity and the ease of causing bruising. From the pathophysiological point of view, there would be a polygenic susceptibility combined with hormonal, microvascular and lymphatic disorders that may be partly responsible for the development of lipedema. It is classified according to the distribution of fat into five types and, according to the severity of the disease, in four stages. The diagnosis is eminently clinical and must be differentiated from other diseases that cause an increase in the volume of the extremities, especially lymphedema and obesity. It is important to carry out a study of the lymphatic system functionality when the diagnosis is not clear or for lipedema staging, so the correct interpretation of these results is essential. Treatment is focused on reducing disability and preventing progression, in order to improve quality of life. Liposuction is currently an effective treatment for lipedema, however, the techniques used for lipectomy in lipedema are different from the techniques used for liposuction for cosmetic purposes. Selective techniques that spare the lymphatic vessels have better results reducing fat volume, delaying progression, reducing pain, reducing gait disturbance, and improving quality of life of these patients.

Key words: lipedema; lymphedema; obesity; lipodystrophy; selective liposuction.

\section{Resumen}

El lipedema es una enfermedad común, frecuentemente subdiagnosticada, crónica y progresiva, que genera un gran deterioro en la calidad de vida. Consiste en el depósito anormal de tejido adiposo subcutáneo principalmente en las extremidades inferiores, afectando casi exclusivamente a mujeres. Sus síntomas principales son el dolor, la sensibilidad y la facilidad para producir equimosis. Desde el punto de vista fisiopatológico, existiría una susceptibilidad poligénica combinada con trastornos hormonales, microvasculares y linfáticos que pueden ser en parte responsables del desarrollo del lipedema. Se clasifica, según la distribución de la grasa en cinco tipos y, según la gravedad de la enfermedad, en cuatro etapas. El diagnóstico es eminentemente clínico y se debe diferenciar de otras patologías que producen aumento de volumen de las extremidades, especialmente el linfedema y obesidad. Es importante realizar un estudio funcional del sistema linfático cuando el diagnóstico es dudoso o para la etapificación del lipedema, por lo que la correcta interpretación de estos resultados es fundamental. El tratamiento está enfocado en disminuir la discapacidad y evitar la progresión, con el fin de mejorar la calidad de vida. Actualmente, la liposucción es un tratamiento efectivo para el lipedema, sin embargo, las técnicas empleadas para la lipectomía en el lipedema son diferentes a las técnicas utilizadas para la liposucción con fines estéticos. Las técnicas selectivas que respetan los vasos linfáticos tienen mejor rendimiento para reducir el volumen de grasa, retrasar la progresión, reducir el dolor, reducir la alteración marcha y mejorar la calidad de vida en estos pacientes.

Palabras clave: lipedema; linfedema; obesidad; lipodistrofia; liposucción selectiva. 


\section{Introducción}

El lipedema es una enfermedad crónica y progresiva que puede provocar una discapacidad considerable, un deterioro del funcionamiento diario y angustia psicosocial ${ }^{1}$. Consiste en el depósito anormal de tejido adiposo subcutáneo, lo que conduce a un aumento bilateral y desproporcionado del volumen de las extremidades inferiores $y$, en algunos casos, extremidades superiores; sin comprometer pies, manos ni tronco. Afecta casi exclusivamente a las mujeres, comenzando con mayor frecuencia entre la pubertad y la tercera década de la vida ${ }^{2}$. A diferencia de la obesidad, los depósitos de grasa y el edema asociados al lipedema son resistentes a los cambios en la dieta, la restricción de la ingesta calórica, la actividad física o la cirugía bariátrica ${ }^{3}$. Los pacientes refieren, además, dolor en las extremidades inferiores que aumenta durante el día, con sensibilidad, facilidad de producción de equimosis e incluso, pudiendo llegar a linfedema progresivo ${ }^{4}$.

La literatura ha mostrado escasos y contradictorios datos sobre la prevalencia del lipedema, pudiendo afectar a millones de mujeres solo en los Estados Unidos, con una incidencia estimada de 1 de cada 9 mujeres adultas ${ }^{5}$. Se estima que entre el $8 \%$ y el $17 \%$ de las mujeres adultas en todo el mundo se ven afectadas, sin embargo, se diagnostica con frecuencia de manera incorrecta y se encuentra subrepresentada en la literatura médica ${ }^{6}$.

\section{Fisiopatología}

Existen muchas interrogantes respecto a la fisiopatología del lipedema, sin embargo, algunos estudios sugieren que existiría una susceptibilidad poligénica combinada con trastornos hormonales, microvasculares y linfáticos que pueden ser en parte responsables de su desarrollo. Existen varios genes, bajo influencia estrogénica, implicados en la angio y linfangiogénesis que pueden desempeñar un papel en la disfunción endotelial y la linfangiopatía?

Desde el punto de vista histológico, el aumento de volumen inicial en el lipedema es producto de una hipertrofia e hiperplasia de los adipocitos ${ }^{8}$. Además del aumento de tamaño de los adipocitos, hay un engrosamiento del intersticio con un incremento del líquido intersticial secundario a una presión hidrostática elevada ${ }^{9,10}$. Si bien, existe un aumento del líquido intersticial, al menos en las primeras etapas el sistema linfático es capaz de funcionar normalmente $^{11}$, por lo que el edema en fases iniciales es probablemente secundario a una sobrecarga del sistema de drenaje, más que una verdadera disfunción del transporte linfático. Sin embargo, a medida que el lipedema progresa, los canales linfáticos se elongan y dilatan desarrollando "microaneurismas" que tienden a filtrar, lo cual se ha podido evidenciar en estudios con linforresonancia ${ }^{12}$. Estos microaneurismas, sumado al aumento del líquido intersticial, conducen a lipolinfedema en una etapa tardía ${ }^{11,13}$. Un estudio de Bilancini et al. ${ }^{14}$, demostró un patrón linfo-cintigráfico anormal con disminución del flujo linfático en 12 mujeres con lipedema, similar a lo observado en pacientes con linfedema primario.

Además de la hipertrofia de los adipocitos, el engrosamiento intersticial y los cambios linfáticos, el plexo vascular subdérmico también presenta cambios estructurales compatibles con microangiopatía. Esta alteración produce fragilidad capilar y filtración, lo que explica la facilidad en la aparición de equimosis y telangiectasias en pacientes con lipede$\mathrm{ma}^{15,16}$. La microangiopatía puede ser consecuencia de una disfunción endotelial primaria a través de un mecanismo de hipoxia, con el consiguiente aumento de la fragilidad vascular similar al observado en pacientes con retinopatía diabética ${ }^{4}$. Además, se han encontrado niveles plasmáticos elevados de factor de crecimiento vascular endotelial (VEGF) en pacientes con lipedema, lo que sugiere un rol de la angiogénesis patológica en el desarrollo de la enfermedad. Por lo tanto, la angiogénesis y el aumento de la permeabilidad capilar pueden ser consecuencias de una adipogénesis desregulada, lo que lleva a una expansión anormal de la grasa y a una posterior hipoxia tisular ${ }^{17}$.

Recientemente se reportó que pacientes con lipedema sin obesidad concomitante, mostraban adipocitos hipertróficos, un mayor número de macrófagos y vasos sanguíneos, y dilatación de capilares en la grasa del muslo en comparación con los controles $\operatorname{sanos}^{18}$. Tales hallazgos sugieren que, en el lipedema, la inflamación y la angiogénesis pueden ocurrir independientemente de la obesidad, respaldando el rol de una microcirculación alterada en la manifestación de la enfermedad.

\section{Presentación clínica y diagnóstico}

Tradicionalmente, la distribución de la grasa en el lipedema es de tipo ginecoide afectando principalmente las caderas, glúteos, muslos y piernas, lo que resulta en una desproporción entre la parte superior e inferior del cuerpo (relación cintura:cadera $<1$ ).

A menudo se confunde el lipedema con la obesidad. En el lipedema, un sello distintivo es la 


\section{CIRUGíA AL DÍA}

Tabla 1. Diferencias clínicas entre lipedema, linfedema y obesidad

\begin{tabular}{|llll|}
\hline & Lipedema & Linfedema & Obesidad \\
Sexo & Mujeres & Mujeres y hombres & Mujeres y hombres \\
Localización & Extremidades bilateral & Extremidades uni o bilateral & Generalizada \\
Edema & $\begin{array}{l}\text { Sin fóvea, cambios mínimos con } \\
\text { elevación y compresión }\end{array}$ & $\begin{array}{l}\text { Con fóvea, se reduce con elevación y } \\
\text { compresión }\end{array}$ & $\begin{array}{l}\text { Sin edema, sin variación con elevación } \\
\text { Equimosis }\end{array}$ \\
Frecuente y fácil & No característico & No característico \\
Turgor & Blando & Firme & Blando \\
Dolor & Sensible a la palpación & No sensible & No característico \\
Infeccón & Infrecuente & Frecuente & Infrecuente \\
\hline
\end{tabular}

Tabla 2. Clasificación del lipedema según la distribución de la grasa (tipos de lipedema)

\begin{tabular}{|ll|}
\hline Tipo I & Pelvis, glúteos y caderas \\
Tipo II & Desde caderas a rodillas, con pliegues en la cara interna de la rodilla \\
Tipo III & Desde caderas a tobillos \\
Tipo IV & Brazos \\
Tipo V & Piernas aisladas \\
\hline
\end{tabular}

Modificado de Plast Reconstr Surg Glob Open 2016;4:e1043.

discrepancia significativa en la adiposidad de las extremidades en comparación con el tronco, mientras que la adiposidad asociada con la obesidad inducida por el estilo de vida es más bien generalizada y proporcionada. Del mismo modo, a pesar de un elevado índice de masa corporal, la incidencia de diabetes es relativamente baja entre las mujeres con lipedema ${ }^{19}$. Otro error frecuente es confundir el lipedema con el linfedema. Aunque la disfunción linfática es un hallazgo común en etapas avanzadas del lipedema, existen muchas características que diferencian el lipedema del linfedema (Tabla 1). Una característica distintiva del lipedema es una diferencia abrupta entre el tejido normal y anormal a nivel del tobillo o muñeca ("signo del manguito") y una desproporción significativa en la circunferencia entre las caderas y la cintura. Por otro lado, las mujeres con lipedema a menudo pueden usar calzado regular y pueden pellizcar la piel en el dorso de sus pies y manos sin dificultad (signo de Kaposi-Stemmer "negativo") ${ }^{20}$.

Según la distribución de la grasa, se han descrito cinco tipos de lipedema, siendo más frecuentes los tres primeros (Tabla 2) ${ }^{21}$. En el tipo I, el tejido graso del lipedema se acumula alrededor de las caderas y los glúteos; en el tipo II, el compromiso es desde las caderas hasta las rodillas; y en el tipo III, se observa un fenotipo de caderas a tobillos. Aproximadamente el $80 \%$ de las mujeres afectadas tienen un compromiso adicional de los brazos (tipo IV), mientras que es poco frecuente el compromiso exclusivo a nivel de las pantorrillas (tipo $\mathrm{V}$ ).

El lipedema tiene una tendencia a progresar en el tiempo, de modo que la gravedad de la enfermedad puede etapificarse. Actualmente existen 4 etapas de lipedema (Figura 1): la etapa 1 consiste en una piel lisa y uniforme, pero con una hipodermis engrosada; en la etapa 2 se evidencia un patrón de piel irregular con el desarrollo de nódulos subcutáneos palpables, lipomas y/o angiolipomas (piel de naranja); la etapa 3 incluye la formación de pliegues de grasa nodular que causan una deformidad severa del contorno de los muslos y alrededor de las rodillas; y la etapa 4 está definida por el desarrollo de linfedema concomitante $^{22}$. El lipolinfedema es el resultado de la progresión a largo plazo de un linfedema inicialmente subclínico, que representa una etapa más avanzada. Además del edema de los pies, con un signo de Stemmer positivo patognomónico, la etapa 4 puede ir acompañada de otras características comunes del linfedema avanzado, como celulitis a repetición o papilomatosis. Generalmente, la progresión de la enfermedad es heterogénea y muy variable, ya que algunas mujeres desarrollan lipedema leve y estable en el tiempo, mientras que otras muestran una progresión gradual con exacerbación repentina inducida por factores estresores (por ejemplo, embarazo o cirugías) ${ }^{23}$.

Clínicamente las pacientes con lipedema refieren facilidad para producir equimosis y dolor importante a la presión de las extremidades afectadas. Sin embargo, la mayoría también experimenta dolor espontáneo. El edema ortostático es otro signo cardinal, que puede ser responsable de la sensación 


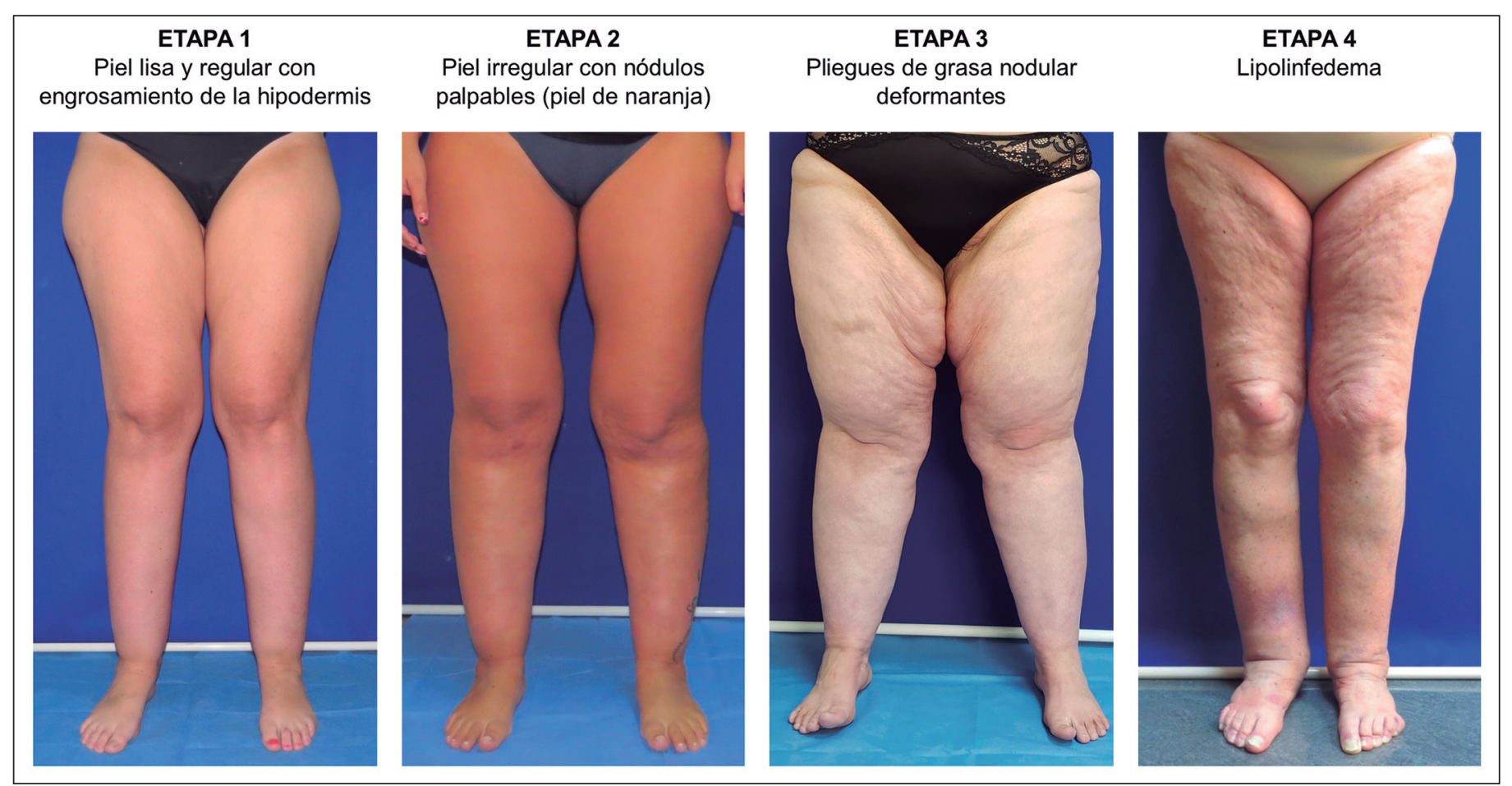

Figura 1. Etapificación del lipedema de acuerdo a su gravedad (etapas del lipedema).

de pesadez, fatiga e incomodidad de las piernas. Este edema y el dolor empeoran con el calor y el ejercicio, y no se alivian con la elevación de las extremidades. Por otra parte, las medidas para bajar de peso tienen un mínimo efecto sobre la distribución anormal de la grasa corporal en pacientes con lipedema. Esto puede conducir a trastornos alimentarios, a un mayor riesgo de depresión y otros problemas psicológicas que comúnmente están asociados a esta enfermedad ${ }^{19}$.

El exceso de grasa en glúteos, caderas, muslos y la parte inferior de las piernas puede afectar la marcha con el consiguiente mal alineamiento del eje de las piernas, lo que genera sobrecarga en las articulaciones $^{24}$. Esto puede provocar osteoartritis de rodilla en valgo, marcha antálgica y sobrepronación de los pies. Además, se pueden producir lesiones cutáneas, maceración e infección debido al exceso de tejido y a los pliegues profundos en la piel.

El diagnóstico de lipedema es eminentemente clínico y se basa en ciertos criterios, habiendo excluido previamente otras enfermedades (Tabla $3)^{21}$. El diagnóstico es altamente probable cuando están presentes los criterios: A (1 al 6) + $(\mathrm{B}[1+2]$ o C [1 + 2] o D [1 + 2] o E). En ausencia de, como máximo, dos de estos criterios (A a E), la presencia de los criterios adicionales F (1) o F (2) también respaldan el diagnóstico.

El diagnóstico diferencial del lipedema se hace con afecciones que se presentan con edema o lipodistrofia de las extremidades inferiores, representadas principalmente por linfedema y obesidad (Tabla 1) ${ }^{25-27}$. También se deben considerar y descartar otras causas de edema clásicas como insuficiencia venosa crónica, edema cíclico idiopático, insuficiencia cardíaca, hepática o renal, mixedema y edema ortostático. En el linfedema, la piel suele estar engrosada a diferencia de lo que sucede en el lipedema, sin embargo, muchas veces distinguir el lipedema de linfedema puede ser difícil porque ambas condiciones pueden coexistir en etapas avanzadas de la enfermedad. A diferencia del linfedema, en el lipedema la presión bimanual generalmente induce dolor.

Desde el punto de vista del imagenológico, es importante realizar un estudio funcional del sistema linfático mediante linfo-cintigrafía y/o linfografía con verde de indocianina, ya que pueden mostrar un drenaje linfático deteriorado en las extremidades con lipedema. Generalmente, este deterioro es menos avanzado que en pacientes con linfedema, pudiendo evidenciar disfunción linfática en etapa subclínica ${ }^{28}$. 
Tabla 3. Criterios diagnósticos de lipedema

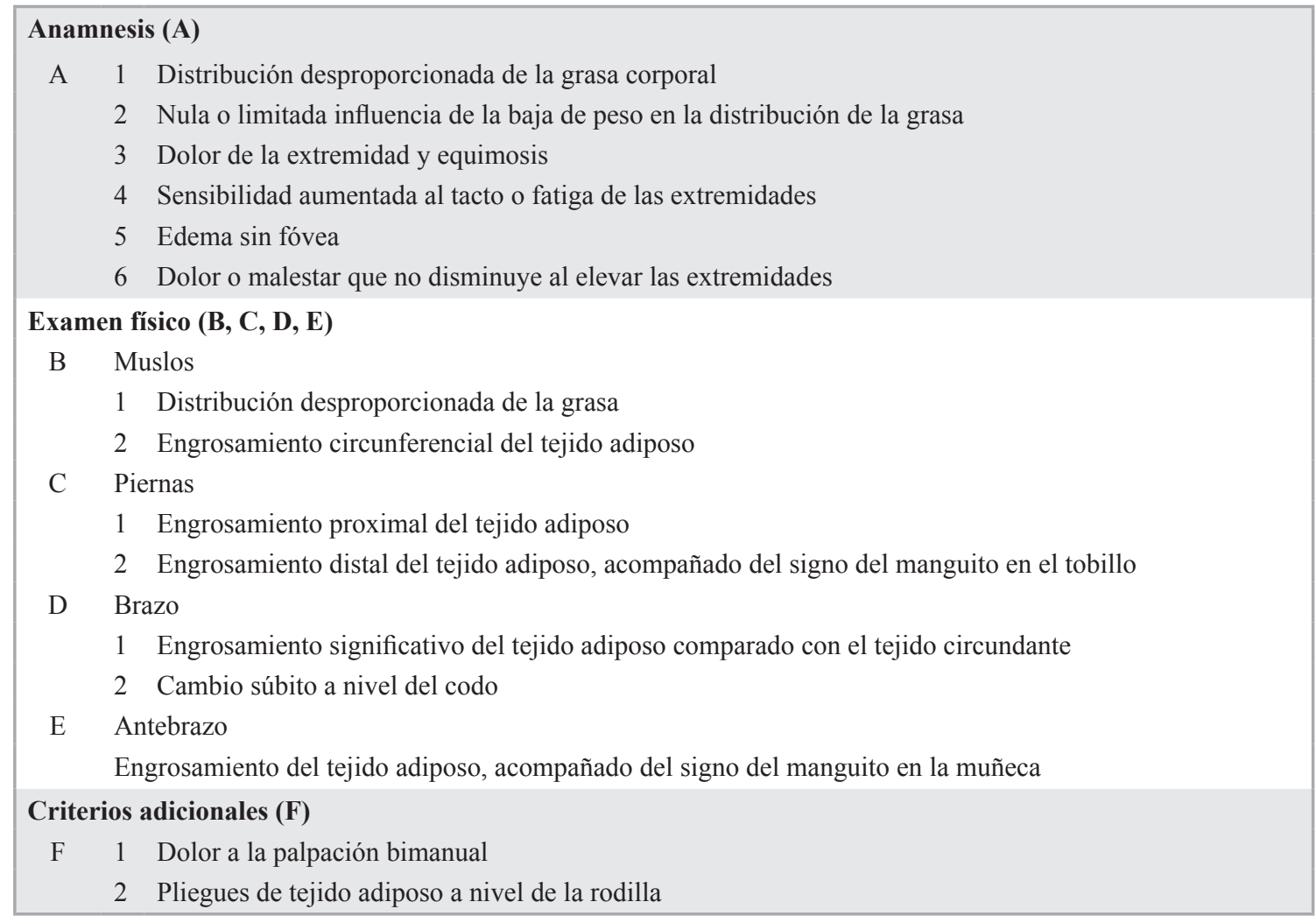

Modificado de Obesity 2019;27:1567-76. *El diagnóstico es altamente probable cuando están presentes los criterios: $\mathrm{A}(1 \mathrm{al} 6)+(\mathrm{B}[1+2]$ o $\mathrm{C}[1+2]$ o D [1 + 2] o E). En ausencia de, como máximo, dos de estos criterios (A a E), la presencia de los criterios adicionales $\mathrm{F}(1)$ o F (2) también respaldan el diagnóstico.

A la luz de estos hallazgos, las técnicas de imagen pueden considerarse una herramienta útil cuando el diagnóstico es dudoso o para la etapificación del lipedema, por lo que su correcta interpretación es fundamental. La resonancia magnética, la tomografia computarizada y la ecografía cutánea de alta resolución también se han utilizado para realizar el diagnóstico diferencial con linfedema, sin embargo, se utilizan con menor frecuencia en la práctica clínica ${ }^{29}$.

Debido a sus similitudes, el lipedema se puede considerar como una enfermedad del espectro de los trastornos adiposos raros o trastornos del tejido adiposo, que incluye la lipomatosis familiar múltiple, la enfermedad de Madelung y enfermedad de Dercum $^{19}$. La enfermedad de Dercum (adiposis dolorosa) es una afección que tiene cierta superposición con el lipedema, ya que ambos comparten características cardinales como dolor y equimosis espontáneas o inducidos por la palpación. Al comienzo, la enfermedad de Dercum se caracteriza por múltiples lipomas dolorosos con posible progresión a depósito graso difuso circunscrito o generalizado; suele acompañarse de cefalea y depresión recurrente, que se describen con menor frecuencia en el lipedema ${ }^{30}$. En la actualidad, se desconoce si cada una de estas enfermedades representa un trastorno único o, más bien, una variación de un fenómeno molecular subyacente común que produce hipertrofia e hiperplasia de adipocitos. Desde el punto de vista clínico, el lipedema se puede distinguir de estas enfermedades debido a la distribución característica del tejido adiposo; respetando la cara, el cuello y el tronco; sin que predomine la presencia de lipomas en el tejido subcutáneo como en los otros trastornos adiposos $\operatorname{raros}^{19}$.

\section{Tratamiento}

El tratamiento del lipedema puede ser conservador o quirúrgico, siendo el objetivo principal 
disminuir la discapacidad, evitar la progresión y prevenir la aparición de complicaciones, con el fin de mejorar la calidad de vida.

Dada la relación que existe entre la disfunción linfática y la hipertrofia de adipocitos que puede progresar a lipolinfedema, se infiere que los tratamientos destinados a mejorar el drenaje linfático complementan el manejo del lipedema. Aunque ha sido controversial el rol de la terapia descongestiva compleja, se ha reportado una mejoría en el edema, en la aparición de petequias y en la fragilidad capilar ${ }^{31}$. Si bien, la grasa del lipedema es resistente a los cambios en el estilo de vida, hay evidencia que apoya los beneficios del ejercicio físico, especialmente los que se realizan en el agua ${ }^{19}$. Por otro lado, el apoyo psicosocial para las pacientes con lipedema es muy importante, ya que con frecuencia presentan afecciones como depresión, ansiedad y trastornos alimentarios.

Desde el punto de vista quirúrgico, el método menos invasivo para eliminar la grasa del lipedema es la liposucción. Sin embargo, es importante recalcar que las técnicas empleadas para la lipectomía en el lipedema son diferentes a las técnicas utilizadas para la liposucción con fines estéticos ${ }^{32-35}$. Específicamente, las técnicas empleadas para la liposucción del lipedema utilizan dispositivos que eliminan la grasa de una manera menos traumática, como la liposucción asistida por potencia (powerassisted liposuction - PAL) con una cánula vibratoria o la liposucción asistida por agua (water-assisted liposuction - WAL). En PAL, el aislamiento de los adipocitos se produce como resultado de la variación de la inercia del tejido adiposo y conectivo producto de la vibración. En $W A L$ las células adiposas se movilizan de manera comparable sin causar mayor perjuicio ${ }^{32}$. Se ha reportado que estos métodos pueden producir menos daño en los vasos linfáticos que las técnicas tradicionales $\mathrm{y}$, por 10 tanto, ser técnicas de liposucción más selectivas ya que respetan la anatomía del sistema linfático sin producir más daño ${ }^{32}$. En un estudio recientemente publicado se evidenció que la liposucción en el lipedema no deteriora la función linfática, es más, se observó una leve mejoría demostrando que es un procedimiento seguro ${ }^{36}$.

Los cirujanos plásticos que nos dedicamos a tratar esta patología, para la liposucción selectiva utilizamos cánulas especializadas que se deben orientar en sentido longitudinal al eje de los vasos linfáticos dentro del tejido adiposo del lipedema, de manera de minimizar posibles lesiones linfáticas a este nivel. Diversos reportes detallan la efectivi- dad de la liposucción para el tratamiento del lipedema con sus técnicas específicas, lo cual no solo han servido para reducir el volumen de grasa del lipedema, sino también para retrasar la progresión de la enfermedad, reducir el dolor de lipedema, reducir la alteración de la marcha y la limitación de la movilidad, y mejorar la calidad de vida en estos pacientes utilizando diversas escalas de medición $^{32-34}$. Es más, se ha visto que los resultados son mejores y más duraderos si los pacientes se operan en etapas más precoces de la enfermedad ${ }^{37}$. En el último Consenso Internacional de Lipedema, se concluyó que, actualmente, la liposucción respetando los vasos linfáticos es el único tratamiento efectivo para el lipedema ${ }^{38}$.

En un reciente estudio, Bauer et al. ${ }^{39}$, reportaron que la mayoría de los pacientes requirieron al menos cuatro cirugías para lograr resultados satisfactorios, entre los que se mencionan disminución significativa del dolor, del edema y equimosis; asimismo e interesantemente, los pacientes que presentaban migraña antes de la liposucción experimentaron una disminución significativa de la frecuencia y/o intensidad de los episodios. En mi experiencia, he logrado evidenciar una mejoría significativa tanto desde el punto de vista funcional como estético, realizando una liposucción selectiva del tejido lipedematoso respetando la anatomía linfática, lo cual mejora significativamente la calidad de vida de las pacientes utilizando LeQOLiS ${ }^{40}$ (Figura 2).

En casos más severos, la cirugía debe ser más extensa mediante la resección longitudinal o tipo tumorectomía de los depósitos de tejido lipedematoso que se acumulan incluso formando pliegues, permitiéndoles a los pacientes una mejoría desde el punto de vista funcional ${ }^{41}$.

Al igual que previo a la cirugía, en el período posoperatorio también es importante continuar con las medidas de apoyo linfático, como la compresión y la terapia descongestiva compleja (rehabilitación kinesiterápica que incluye vendajes, las prendas de compresión, el drenaje linfático manual, el ejercicio y los cuidados personales). Este complemento ayuda a estimular al sistema linfático para disminuir el edema posoperatorio y la solución de infiltración residual. Del mismo modo, también es importante que los pacientes continúen con un estilo de vida saludable, incluyendo buenos hábitos alimentarios y programas de ejercicio. Si bien, estas medidas no necesariamente afectarán en la grasa del lipedema, mejorarán la salud y el bienestar general de los pacientes antes y después de la cirugía. 


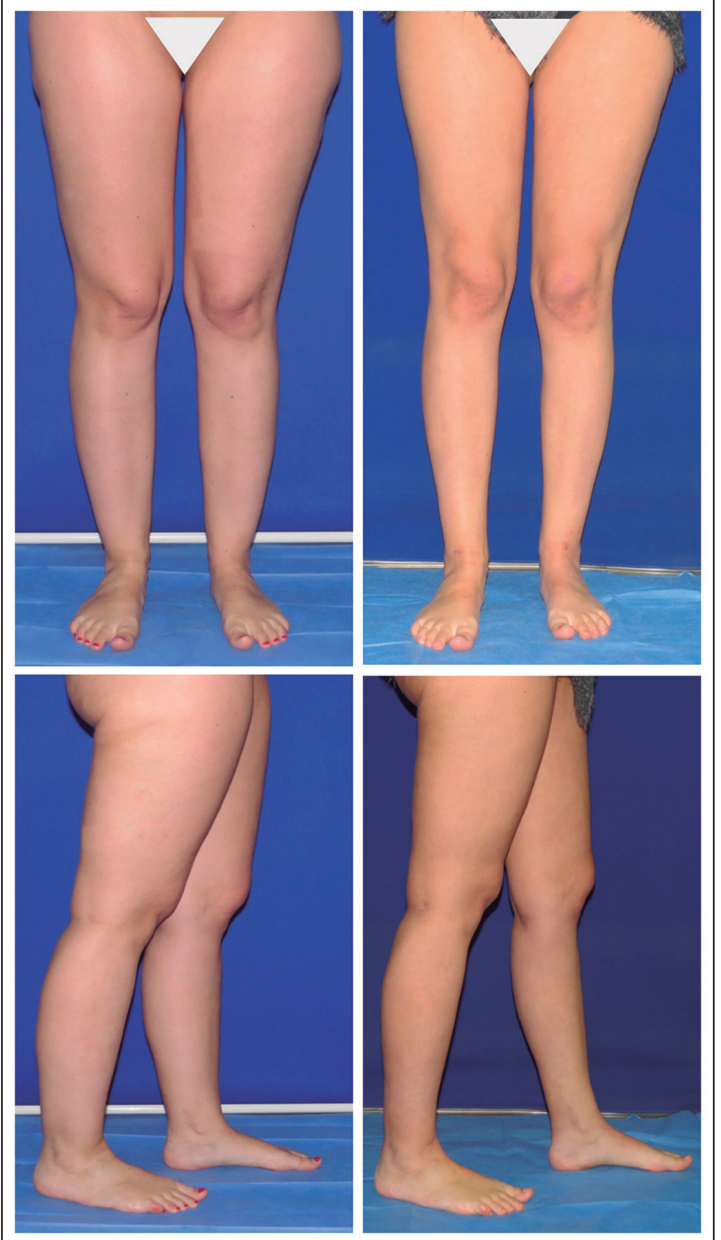

Figura 2. Paciente de 36 años, sexo femenino, con lipedema tipo III/etapa 1. Se realizó liposucción selectiva, presentando una disminución significativa de los síntomas y un excelente resultado estético. A la izquierda fotografías previas a la cirugía y a la derecha fotografías 10 meses posoperatorio.

\section{Conclusiones}

El lipedema es una enfermedad común, frecuentemente subdiagnosticada, de carácter crónico y progresivo, que genera un gran deterioro en la calidad de vida de las pacientes que lo padecen. Su diagnóstico es eminentemente clínico, habiendo descartado otras patologías y haciendo el diagnóstico diferencial con ciertas enfermedades como el linfedema y la obesidad. La caracterización de las pacientes y un estudio de la funcionalidad linfática pueden orientar al diagnóstico y al tipo de tratamiento a indicar. El tratamiento quirúrgico de elección es la liposucción selectiva respetando los vasos linfáticos, el cual ha demostrado una reducción significativa del volumen, una mejoría sintomática $y$, además, beneficios desde el punto de vista estético, lo cual mejora la calidad de vida. Es importante generar conciencia sobre esta enfermedad, realizar investigaciones adicionales e identificar mejores modalidades diagnósticas y terapéuticas para que las mujeres que presentan lipedema puedan beneficiarse de un tratamiento óptimo.

\section{Responsabilidades éticas}

Protección de personas y animales. Los autores declaran que para esta investigación no se han realizado experimentos en seres humanos ni en animales.

Confidencialidad de los datos. Los autores declaran que en este artículo no aparecen datos de pacientes.

Conflictos de interés: no hay.

\section{Bibliografía}

1. Forner-Cordero I, Szolnoky G, FornerCordero A, Kemény L. Lipedema an overview of its clinical manifestations, diagnosis and treatment of the disproportional fatty deposition syndromesystematic review. Clin Obes. 2012;2:8695.

2. Fife CE, Maus EA, Carter MJ. Lipedema: a frequently misdiagnosed and misunderstood fatty deposition syndrome. Adv Skin Wound Care 2010;23:81-92; quiz 93-4.

3. Warren Peled A, Kappos EA. Lipedema:
Diagnostic and management challenges. Int J Womens Health 2016;8:389-95.

4. Wenczl E, Daróczy J. Lipedema, a barely known disease: Diagnosis, associated diseases and therapy. Orv Hetil. 2008;149:2121-7.

5. Foldi E, Foldi M. Lipedema. In: Foldi M, Foldi E, eds. Foldi's Textbook of Lymphology. Munich, Germany: Elsevier $\mathrm{GmbH} ; 2006: 417-27$.

6. Buck DW 0II, Herbst KL. Lipedema: A relatively common disease with extremely common misconceptions. Plast Reconstr Surg Glob Open 2016;4:e1043.

7. Szel E, Kemeny L, Groma G, Szolnoky G.
Pathophysiological dilemmas of lipedema. Med Hypotheses 2014;83:599-606.

8. van Geest AJ, Esten SCAM, Cambier J-PRA, Cambier A, Gielen EGJ, Kessels A, et al. Lymphatic disturbances in lipoedema. Phlebologie 2003;32:138-42.

9. Stallworth JM, Hennigar GR, Jonsson HT Jr, Rodriguez O. The chronically swollen painful extremity. A detailed study for possible etiological factors. JAMA 1974;228:1656-9.

10. Harwood CA, Bull RH, Evans J, Mortimer PS. Lymphatic and venous function in lipoedema. Br J Dermatol. 1996;134:1-6. 
11. Amann-Vesti BR, Franzeck UK, Bollinger A. Microlymphatic aneurysms in patients with lipedema. Lymphology 2001;34:1705.

12. Lohrmann C, Foeldi E, Langer M. MR imaging of the lymphatic system in patients with lipedema and lipo-lymphedema. Microvasc Res. 2009;77:335-9.

13. Bräutigam P, Földi E, Schaiper I, Krause T, Vanscheidt W, Moser E. Analysis of lymphatic drainage in various forms of leg edema using two compartment lymphoscintigraphy. Lymphology 1998;31:43-55.

14. Bilancini S, Lucchi M, Tucci S, Eleuteri P. Functional lymphatic alterations in patients suffering from lipedema. Angiology 1995;46:333-9.

15. Curri SB, Merlen JF. Microvascular disorders of adipose tissue. J Mal Vasc. 1986;11:303-9.

16. Merlen JF, Curri SB, Sarteel AM. Cellulitis, a conjunctive microvascular disease. Phlebologie 1979;32:279-82.

17. Siems W, Grune T, Voss P, Brenke R. Anti-fibrosclerotic effects of shock wave therapy in lipedema and cellulite. BioFactors 2005;24:275-82.

18. Al-Ghadban S, Cromer W, Allen M, Ussery C, Badowski M, Harris D, et al. Dilated blood and lymphatic microvessels, angiogenesis, increased macrophages, and adipocyte hypertrophy in lipedema thigh skin and fat tissue. Journal of Obesity 2019;2019, ArticleID 8747461, 10 pages. https://doi.org/10.1155/2019/8747461

19. Herbst KL. Rare adipose disorders (RADs) masquerading as obesity. Acta Pharmacol Sin. 2012;33:155-72.

20. Wold LE, Hines EA, Jr, Allen EV. Lipedema of the legs; a syndrome characterized by fat legs and edema. Ann Intern Med. 1951;34:1243-50.

21. Buso G, Depairon M, Tomson D, Raffoul W, Vettor R, Mazzolai L. Lipedema: A Call to Action! Obesity 2019;27:1567-76.

22. Torre YS, Wadeea R, Rosas V, Herbst KL.
Lipedema: friend and foe. Horm Mol Biol Clin Investig. 2018;33:1-10.

23. Langendoen SI, Habbema L, Nijsten TE, Neumann HA. Lipoedema: from clinical presentation to therapy. A review of the literature. Br J Dermatol. 2009;161:980-6.

24. Stutz J. Liposuction in lipedema to prevent later joint complications. Vasomed 2011;23:2-6.

25. Pereira N, Koshima I. Linfedema: actualización en el diagnóstico y tratamiento quirúrgico. Rev Chil Cir. 2018;70:589-97.

26. Pereira N, Yamamoto T. Linfedema de extremidades inferiores secundario al tratamiento oncológico: Actualización en el Diagnóstico y Tratamiento Quirúrgico. Rev Cirugía 2019;71:88-97.

27. Pereira N, Pons G, Masià J. Linfedema Asociado al Cáncer de Mama: Factores de Riesgo, Diagnóstico y Tratamiento Quirúrgico. Rev Cirugía 2019;71:79-87.

28. Boursier V, Pecking A, Vignes

S. Comparative analysis of lymphoscintigraphy between lipedema and lower limb lymphedema. J Mal Vasc. 2004;29:257-61.

29. Iker E, Mayfield CK, Gould DJ, Patel KM. Characterizing lower extremity lymphedema and lipedema with cutaneous ultrasonography and an objective computer assisted measurement of dermal echogenicity. Lymphat Res Biol. 2019;17:525-30.

30. Brodovsky S, Westreich M, Leibowitz A, Schwartz Y. Adiposis dolorosa (Dercum's disease): 10-year follow-up. Ann Plast Surg. 1994;33:664-8.

31. Szolnoky G, Nagy N, Kovács RK, Dósa-Rácz E, Szabó A, Bársony K, et al. Complex decongestive physiotherapy decreases capillary fragility in lipedema. Lymphology 2008;41:161-6.

32. Stutz JJ, Krahl D. Water jet-assisted liposuction for patients with lipoedema: histologic and immunohistologic analysis of the aspirates of 30 lipoedema patients. Aesthetic Plast Surg. 2009;33:153-62.
33. Rapprich S, Dingler A, Podda M. Liposuction is an effective treatment for lipedema-results of a study with 25 patients. J Dtsch Dermatol Ges. 2011;9:3340.

34. Rapprich S, Baum S, Kaak I, Kottmann T, Podda M. Treatment of lipoedema using liposuction. Results of our own surveys. Phlebologie 2015;3:1-13.

35. Schmeller W, Hueppe M, Meier-Vollrath I. Tumescent liposuction in lipoedema yields good long-term results. Br J Dermatol. 2012;166:161-8.

36. van de Pas CB, Boonen RSM, Stevens S, Willemsen S, Valkema R, Neumann M. Does Tumescent Liposuction Damage the Lymph Vessels in Lipoedema Patients? Phlebology 2020;35:231-6.

37. Dadras M, Mallinger PJ, Corterier CC, Theodosiadi S, Ghods M. Liposuction in the Treatment of Lipedema: A Longitudinal Study. Arch Plast Surg. 2017;44:324-31.

38. Sandhofer M, Hanke CW, Habbema L, Podda M, Rapprich S, Schmeller W, et al. Prevention of Progression of Lipedema With Liposuction Using Tumescent Local Anesthesia: Results of an International Consensus Conference. Dermatol Surg. 2020;46:220-8.

39. Bauer AT, von Lukowicz D, Lossagk K, Aitzetmueller M, Moog P, Cerny M, et al. New Insights on Lipedema: The Enigmatic Disease of the Peripheral Fat. Plast Reconstr Surg. 2019;144:147584.

40. Pereira N, Venegas J, Cifuentes I. Calidad de vida en pacientes sometidos al tratamiento quirúrgico del linfedema. Validación lingüística y adaptación transcultural del Lymphedema Quality of Life Score (LeQOLiS). Rev. Cirugía 2020;72:113-7.

41. Halk AB, Damstra RJ. First Dutch guidelines on lipedema using the international classification of functioning, disability and health. Phlebology 2016;32:152-9. 